\title{
Molecular characterization of aquaglyceroporine: a novel mutation in LMAQP1 from LEISHMANIA MAJOR (MRHO/IR/75/ER)
}

\author{
G. Eslami*, M. Ghavami \\ Research Center for Food Hygiene and Safety, Shahid Sadoughi University of Medical Sciences, Yazd, Iran \\ *e-mail: eslami_g2000@yahoo.com
}

Key words: Aquaporin 1, Leishmania, Molecular Dynamics Simulation, Antimony

Motivation and Aims: The first line treatment for cutaneous leishmaniasis is pentavalent antimony such as sodium stibogluconate (pentostam) and meglumine antimonite (glucantime). One of the most important way to uptake the drug is by a transmembrane protein, called aquaglyceroporin encoded by Aquaglyceroprotein1 (LmAQP1). So far, there is no report on $L m A Q P 1$ from L. major (MRHO/IR/75/ER), therefore, in this study, molecular characterization of $L m A Q P 1$ was reported.

Methods and Algorithms: L. major (MRHO/IR/75/ER) promastigotes were cultured, and then DNA extraction and RNA extraction were done and followed by cDNA synthesis. Amplicons resulted from PCR and RT-PCR using specific primers were purified and sequenced. Molecular characterization was done by bioinformatically softwares such as BLAST, ClustalW2, and RMSD.

Results: Amplicons resulted from PCR and RT-PCR showed equal size in length. BLASTn analysis showed a point nucleotide change in LmAQP1 gene that encoded 282-amino-acid long protein with a mutation at position 154 including replacement of alanine by threonine. The observed mutation in the interested gene was assessed using the above mentioned software. The mentioned gene was submitted at GenBank, NCBI with accession number of KU514052.

Conclusion: The functional prediction of the protein encoded from LmAQP1 showed that the mentioned mutation could not affect the three dimension structure, but it may modify the drug uptake potential of this important channel. Based on from LmAQPI role, it seems to be an appropriate candidate for drug development. According to search through internet, this is the first report of $L m A Q P 1$ from L. major (MRHO/IR/75/ER). Availability: The mentioned strain is available in esearch Center for Food Hygiene and Safety, Shahid Sadoughi University of Medical Sciences, Yazd, Iran.

\section{References}

1. Marquis N. et al. (2005) Modulation in aquaglyceroporin AQP1 gene transcript levels in drug-resistant Leishmania. Mol. Microbiol. 57:1690-1699.

2. Figarella K. et al. (2007) Biochemical characterization of Leishmania major aquaglyceroporin LmAQP1: possible role in volume regulation and osmotaxis. Mol. Microbiol. 65:1006-1017.

3. Mukhopadhyay R. et al. (2011) The role of alanine 163 in solute permeability of Leishmania major aquaglyceroporin LmAQP1, Mol. Biochem. Parasitol. 175:83-90.

4. Monte-Neto R. et al. (2015) Intrachromosomal amplification, locus deletion and point mutation in the aquaglyceroporin AQP1 gene in antimony resistant Leishmania (Viannia) guyanensis. PLoS Negl Trop Dis. 9:e003476. 